\begin{tabular}{|c|l|}
\hline Title & Singularities of lightcone Gauss images of spacelike hy persurfaces in de Sitter space \\
\hline Author(s) & Kasedou, Masaki \\
\hline Citation & Hokkaido University Preprint Series in Mathematics, 911, 1-17 \\
\hline Issue Date & 2008 \\
\hline DOI & 10.14943/84061 \\
\hline Doc URL & http://hdl.handle.net/2115/69719 \\
\hline Type & bulletin (article) \\
\hline File Information & pre911.pdf \\
\hline
\end{tabular}

Instructions for use 


\title{
Singularities of lightcone Gauss images of spacelike hypersurfaces in de Sitter space
}

\author{
Masaki Kasedou
}

\begin{abstract}
We define the notions of lightcone Gauss images of spacelike hypersurfaces in de Sitter space. We investigate the relationships between singularities of these maps and geometric properties of spacelike hypersurfaces as an application of the theory of Legendrian singularities. We classify the singularities and give some examples in the generic case in de Sitter 3-space.

Mathematics Subject Classification (2000): 53A35, 53B30, 58C25.

Key words: de Sitter space, Gauss image, spacelike hypersurface, lightcone.
\end{abstract}

\section{Introduction}

In this paper, we discuss the extrinsic differential geometry of the spacelike hypersurfaces in de Sitter space. Bleeker and Wilson [3] studied the singularities of the Gauss map of a surface in Euclidean 3-space. In their paper, the main theorem asserts that the generic singularities of Gauss maps are folds or cusps. Banchoff et al. [2], Landis [6] and Platnova [8] studied geometric meanings of cusps of the Gauss map of a surface. Bruce [4] and Romero-Fuster [9] have also independently studied the singularities of the Gauss map and the dual of hypersurface in Euclidean space. The main tool of Bruce and Romero-Fuster for the study is the family of height functions on a hypersurface. Izumiya et al. [5] studied the extrinsic differential geometry on hypersurfaces in hyperbolic space as an application of the theory of Legendrian singularities.

The investigation in this paper is the analogue of that in [5] for spacelike hypersurfaces in de Sitter space. It is known that de Sitter space is a Lorentzian space form with positive curvature which is one of the vacuum solutions of the Einstein equations. In $\S 2$ we introduce the notion of the lightcone Gauss image and the lightcone Gauss-Kronecker curvature. The lightcone GaussKronecker curvature is a invariant under the Lorentzian transformation in de Sitter space. In $\S 3,4$ we introduce a family of functions that is called the lightcone height function on the spacelike hypersurface. The singular set of the lightcone Gauss image is the lightcone parabolic set of the spacelike hypersurface and this can be interpreted as the discriminant set of the family of height functions. In $\S 5,6$ we discuss the contact between hypersurfaces and de Sitter hyperhorospheres. We apply the theory of Legendrian singularities for the study of lightcone Gauss images of generic hypersurfaces. In $\S 8$ we classify the singularities of lightcone Gauss images of generic spacelike surfaces in de Sitter 3-space. Here, we have two singularity types 
of lightcone Gauss images, which are cuspidal edges and swallowtails. In $\S 7,9$ we construct the spacelike Monge form in de Sitter space. It makes us to give examples which are corresponding to generic singularities of lightcone Gauss images.

\section{Hypersurfaces in de Sitter space}

In this section we introduce the local differential geometry in the explicit way. Let $\mathbb{R}^{n+1}=\{\mathbf{x}=$ $\left.\left(x_{0}, \cdots, x_{n}\right) \mid x_{i} \in \mathbb{R}(i=0, \cdots, n)\right\}$ be an $(n+1)$-dimensional vector space. For any vectors $\mathbf{x}=\left(x_{0}, \cdots, x_{n}\right), \mathbf{y}=\left(y_{0}, \cdots, y_{n}\right)$ in $\mathbb{R}^{n+1}$, the pseudo scalar product of $\mathbf{x}$ and $\mathbf{y}$ is defined by

$$
\langle\mathbf{x}, \mathbf{y}\rangle=-x_{0} y_{0}+\sum_{i=1}^{n} x_{i} y_{i}
$$

We call $\left(\mathbb{R}^{n+1},\langle\rangle,\right)$ a Minkowski $(n+1)$-space and write $\mathbb{R}_{1}^{n+1}$ instead of $\left(\mathbb{R}^{n+1},\langle\rangle,\right)$.

We say that a vector $\mathbf{x} \in \mathbb{R}_{1}^{n+1} \backslash\{\mathbf{0}\}$ is spacelike, timelike or lightlike if $\langle\mathbf{x}, \mathbf{x}\rangle>0,\langle\mathbf{x}, \mathbf{x}\rangle=0$ or $\langle\mathbf{x}, \mathbf{x}\rangle<0$ respectively. The norm of the vector $\mathbf{x} \in \mathbb{R}_{1}^{n+1}$ is defined by $\|x\|=\sqrt{|\langle\mathbf{x}, \mathbf{x}\rangle|}$.

For a vector $\mathbf{v} \in \mathbb{R}_{1}^{n+1} \backslash\{\mathbf{0}\}$ and a real number $c$, we define a hyperplane with pseudo-normal $\mathbf{v}$ by

$$
\operatorname{HP}(\mathbf{v}, c)=\left\{\mathbf{x} \in \mathbb{R}_{1}^{n+1} \mid\langle\mathbf{x}, \mathbf{v}\rangle=c\right\} .
$$

We call $\operatorname{HP}(\mathbf{v}, c)$ a spacelike hyperplane, timelike hyperplane or lightlike hyperplane if $\mathbf{v}$ is timelike, spacelike or lightlike respectively.

We now define hyperbolic $n$-space by

$$
H_{+}^{n}(-1)=\left\{\mathbf{x} \in \mathbb{R}_{1}^{n+1} \mid\langle\mathbf{x}, \mathbf{x}\rangle=-1, x_{0} \geq 1\right\}
$$

and de Sitter $n$-space by

$$
S_{1}^{n}=\left\{\mathbf{x} \in \mathbb{R}_{1}^{n+1} \mid\langle\mathbf{x}, \mathbf{x}\rangle=1\right\} .
$$

For any $\mathbf{x}_{1}, \mathbf{x}_{2}, \cdots, \mathbf{x}_{n} \in \mathbb{R}_{1}^{n+1}$, we define a vector $\mathbf{x}_{1} \wedge \mathbf{x}_{2} \wedge \cdots \wedge \mathbf{x}_{n}$ by

$$
\mathbf{x}_{1} \wedge \mathbf{x}_{2} \wedge \cdots \wedge \mathbf{x}_{n}=\left|\begin{array}{cccc}
-\mathbf{e}_{0} & \mathbf{e}_{1} & \cdots & \mathbf{e}_{n} \\
x_{0}^{1} & x_{1}^{1} & \cdots & x_{n}^{1} \\
\vdots & \vdots & \ddots & \vdots \\
x_{0}^{n} & x_{1}^{n} & \cdots & x_{n}^{n}
\end{array}\right|
$$

where $\mathbf{e}_{0}, \mathbf{e}_{1}, \cdots \mathbf{e}_{n}$ are the canonical basis of $\mathbb{R}_{1}^{n+1}$ and $\mathbf{x}_{i}=\left(x_{0}^{i}, x_{1}^{i}, \cdots, x_{n}^{i}\right)$ which satisfies that $\left\langle\mathbf{x}, \mathbf{x}_{1} \wedge \cdots \wedge \mathbf{x}_{n}\right\rangle=\operatorname{det}\left(\mathbf{x}, \mathbf{x}_{1}, \cdots, \mathbf{x}_{n}\right)$, so that $\mathbf{x}_{1} \wedge \cdots \wedge \mathbf{x}_{n}$ is pseudo-orthogonal to any $\mathbf{x}_{i}$ (for $i=1, \cdots, n)$.

We also define a set $L C_{a}=\left\{\mathbf{x} \in \mathbb{R}_{1}^{n+1} \mid\langle\mathbf{x}-\mathbf{a}, \mathbf{x}-\mathbf{a}\rangle\right\}$, which is called a closed lightcone with vertex a. We denote

$$
L C_{ \pm}^{*}=\left\{\mathbf{x}=\left(x_{0}, \cdots, x_{n}\right) \in L C_{\mathbf{0}} \mid x_{0}>0\left(x_{0}<0\right)\right\}
$$


and call it the future (resp. past) lightcone at the origin.

We now study the extrinsic differential geometry of spacelike hypersurfaces in $S_{1}^{n}$. Let $\mathbf{X}: U \longrightarrow S_{1}^{n}$ be an embedding, where $U \subset \mathbb{R}^{n-1}$ is an open subset. We say $\mathbf{X}$ is a spacelike hypersurface in $S_{1}^{n}$ if every non zero vector generated by $\left\{\mathbf{X}_{u_{i}}(u)\right\}_{i=1}^{n-1}$ is always spacelike, where $u=\left(u_{1}, \cdots, u_{n-1}\right)$ is an element of $U$ and $\mathbf{X}_{u_{i}}$ is a partial derivative of $\mathbf{X}$ with respect to $u_{i}$. We denote $M=\mathbf{X}(U)$ and identify $M$ with $U$ through the embedding $\mathbf{X}$. Since $\langle\mathbf{X}, \mathbf{X}\rangle \equiv$ 1, we have $\left\langle\mathbf{X}_{u_{i}}, \mathbf{X}\right\rangle \equiv 0$ (for $i=1, \cdots, n-1$ ). It follows that a hyperplane spanned by $\left\{\mathbf{X}, \mathbf{X}_{u_{1}}, \cdots, \mathbf{X}_{u_{n-1}}\right\}$ is spacelike. We define a vector

$$
\mathbf{e}(u)=\frac{\mathbf{X}(u) \wedge \mathbf{X}_{u_{1}}(u) \wedge \cdots \wedge \mathbf{X}_{u_{n-1}}(u)}{\left\|\mathbf{X}(u) \wedge \mathbf{X}_{u_{1}}(u) \wedge \cdots \wedge \mathbf{X}_{u_{n-1}}(u)\right\|}
$$

Then we have

$$
\left\langle\mathbf{e}, \mathbf{X}_{u_{i}}\right\rangle \equiv\langle\mathbf{e}, \mathbf{X}\rangle \equiv 0, \quad\langle\mathbf{e}, \mathbf{e}\rangle \equiv-1 . \quad(\text { for } i=1, \cdots, n-1),
$$

Therefore the vector $\mathbf{X} \pm \mathbf{e}$ is lightlike. Since $\mathbf{X}(u) \in S_{1}^{n}$ and $\mathbf{e}(u) \in H_{+}^{n}(-1)$, we can show that $\mathbf{X}(u) \pm \mathbf{e}(u) \in L C_{ \pm}^{*}$. We define a map

$$
\mathbb{L}^{ \pm}: U \longrightarrow L C_{ \pm}^{*}
$$

by $\mathbb{L}^{ \pm}(u)=\mathbf{X}(u) \pm \mathbf{e}(u)$, which is called the lightcone Gauss image of $\mathbf{X}$.

We now define the lightcone Gauss-Kronecker curvature and the lightcone mean curvature of the hypersurface $M=\mathbf{X}(U)$. Since $\left\{\mathbf{X}\left(u_{0}\right), \mathbf{e}\left(u_{0}\right), \mathbf{X}_{u_{1}}\left(u_{0}\right), \cdots, \mathbf{X}_{u_{n-1}}\left(u_{0}\right)\right\}$ is a basis of the vector space $T_{p} \mathbb{R}_{1}^{n+1}$, we have the following lemma analogous to ([5], Lemma 2.1).

Lemma 2.1. For any $p=\mathbf{X}\left(u_{0}\right) \in M$ and $\mathbf{v} \in T_{p} M$, we have $D_{\mathbf{v}} \mathbf{e} \in T_{p} M$, so that $D_{\mathbf{v}} \mathbb{L}^{ \pm} \in$ $T_{p} M$. Here, $D_{\mathbf{v}}$ denotes the covariant derivative with respect to the tangent vector $\mathbf{v}$.

We now consider a hypersurface defined by $H P(\mathbf{v}, c) \cap S_{1}^{n}$. We say that $H P(\mathbf{v}, c) \cap S_{1}^{n}$ is an elliptic hyperquadric or a hyperbolic hyperquadric if $H P(\mathbf{v}, c)$ is spacelike or timelike respectively. We say that $H P(\mathbf{v}, 1) \cap S_{1}^{n}$ is a de Sitter hyperhorosphere if $H P(\mathbf{v}, 1)$ is lightlike. Then we have the following proposition.

Proposition 2.2. Let $\mathbf{X}: U \longrightarrow S_{1}^{n}$ be a spacelike hypersurface in $S_{1}^{n}$. The lightcone Gauss image $\mathbb{L}^{ \pm}$is constant if and only if the spacelike hypersurface $M=\mathbf{X}(U)$ is a part of a de Sitter hyperhorosphere.

Proof. Since $\mathbb{L}^{ \pm}(u)$ is constant $\mathbb{L}^{ \pm}$, so we have $\left\langle\mathbf{X}(u), \mathbb{L}^{ \pm}\right\rangle=\langle\mathbf{X}(u), \mathbf{X}(u) \pm \mathbf{e}(u)\rangle=1$ for any $u \in U$. Therefore, we have $\mathbf{X}(U) \subset H P\left(\mathbb{L}^{ \pm},+1\right) \cap S_{1}^{n}$.

If $\mathbf{X}(U) \subset H P(\mathbf{v}, c) \cap S_{1}^{n}$ for some $\mathbf{v} \in L C^{*}$ and $c \neq 0$, then we have $\langle\mathbf{X}(u), \mathbf{v}\rangle=r$ and $\left\langle\mathbf{X}_{u_{i}}(u), \mathbf{v}\right\rangle=\langle\mathbf{v}, \mathbf{v}\rangle=0$. This means that $\mathbf{v}=r \mathbb{L}^{+}(u)$ or $\mathbf{v}=r \mathbb{L}^{+}(u)$. Therefore, $\mathbb{L}^{ \pm}$is a constant vector $(1 / c) \mathbf{v}$.

Under the identification of $U$ and $M$, the derivative $d \mathbf{X}\left(u_{o}\right)$ can be identified with the identity mapping $\operatorname{id}_{T_{p} M}$ on the tangent space $T_{p} M$, where $p=\mathbf{X}\left(u_{o}\right)$. This means that

$$
d \mathbb{L}^{ \pm}\left(u_{0}\right)=\operatorname{id}_{T_{p} M} \pm d \mathbf{e}\left(u_{0}\right) .
$$


By Lemma 2.1, $d \mathbf{e}\left(u_{0}\right)$ is a linear transformation on the tangent space $T_{p} M$, so that $d \mathbb{L}^{ \pm}\left(u_{0}\right)$ is also a linear transformation on $T_{p} M$. We respectively call $S_{p}^{ \pm}=-d \mathbb{L}^{ \pm}\left(u_{0}\right): T_{p} M \longrightarrow T_{p} M$ the lightcone shape operator of $M=\mathbf{X}(U)$ of at $p=\mathbf{X}\left(u_{0}\right)$ and $A_{p}=-d \mathbf{e}: T_{p} M \longrightarrow T_{p} M$ the shape operator of $M=\mathbf{X}(U)$ of at $p=\mathbf{X}\left(u_{0}\right)$. We denote the eigenvalue of $S_{p}^{ \pm}$by $\bar{\kappa}_{p}^{ \pm}$and the eigenvalue of $A_{p}$ by $\kappa_{p}$. By the relation $S_{p}^{ \pm}=-\mathrm{id}_{T_{p} M} \pm A_{p}, S_{p}^{ \pm}$and $A_{p}$ have the common eigenvectors and we have a relation $\bar{\kappa}_{p}^{ \pm}=-1 \pm \kappa_{p}$.

The lightcone Gauss-Kronecker curvature of $M=\mathbf{X}(U)$ at $p=\mathbf{X}\left(u_{0}\right)$ is defined to be

$$
K_{\ell}^{ \pm}\left(u_{0}\right)=\operatorname{det} S_{p}^{ \pm} .
$$

Since $A_{p}$ is the shape operator with respect to the Riemannian metric on $\mathrm{M}$ induced from the Lorentzian metric on $\mathbb{R}_{1}^{n+1}$, we define the Gauss-Kronecker curvature on $M$ by $K\left(u_{0}\right)=\operatorname{det} A_{p}$.

We say that a point $u \in U$ or $p=\mathbf{X}(u)$ is an umbilic point if $S_{p}^{ \pm}=\bar{\kappa}_{p}^{ \pm} \mathrm{id}_{T_{p} M}$. Since the eigenvectors of $S_{p}^{ \pm}$and $A_{p}$ are the same, the above condition is equivalent to the condition $A_{p}=\kappa_{p} \mathrm{id}_{T_{p} M}$. We say that $M=\mathbf{X}(U)$ is totally umbilic if all points on $M$ are umbilic.

Proposition 2.3. Suppose that $M=\mathbf{X}(U)$ is totally umbilic. Then $\bar{\kappa}_{p}^{ \pm}, \kappa_{p}$ are constant $\bar{\kappa}^{ \pm}$, $\kappa$. Under this condition, we have the following classification.

(1) Suppose that $\bar{\kappa}^{ \pm} \neq 0$.

(a) If $0<|\kappa|=\left|\bar{\kappa}^{ \pm}+1\right|<1$, then $M$ is a part of a hyperbolic hyperquadric $H P(\mathbf{v}, 1) \cap S_{1}^{n}$.

(b) If $1<|\kappa|=\left|\bar{\kappa}^{ \pm}+1\right|$, then $M$ is a part of an elliptic hyperquadric $\operatorname{HP}(\mathbf{v}, 1) \cap S_{1}^{n}$.

(c) If $\kappa=\bar{\kappa}^{ \pm}+1=0$, then $M$ is a part of a hyperbolic hyperquadric $\operatorname{HP}(\mathbf{v}, 0) \cap S_{1}^{n}$.

(2) If $\bar{\kappa}^{ \pm}=0$, then $M$ is a part of a de Sitter hyperhorosphere.

Proof. By definition, we have $-\mathbb{L}_{u_{i}}^{ \pm}(u)=\bar{\kappa}_{p}^{ \pm} \mathbf{X}_{u_{i}}(u)$ (for $\left.i=1, \cdots, n-1\right)$ for any $p=\mathbf{X}(u) \in M$. Therefore, we have

$$
\mathbb{L}_{u_{i}, u_{j}}^{ \pm}(u)=\bar{\kappa}_{u_{j} p}^{ \pm} \mathbf{X}_{u_{i}}(u)+\bar{\kappa}_{p}^{ \pm} \mathbf{X}_{u_{i}, u_{j}}(u)
$$

Since $\mathbb{L}_{u_{i}, u_{j}}^{ \pm}=\mathbb{L}_{u_{j}, u_{i}}^{ \pm}$and $\mathbf{X}_{u_{i}, u_{j}}=\mathbf{X}_{u_{j}, u_{i}}$, we have $\bar{\kappa}_{u_{j} p}^{ \pm} \mathbf{X}_{u_{i}}(u)-\bar{\kappa}_{u_{i} p}^{ \pm} \mathbf{X}_{u_{j}}(u)=0$. On the other hand, $\mathbf{X}_{u_{i}}(i=1, \cdots, n-1)$ are linearly independent, so that $\bar{\kappa}_{p}^{ \pm}$is constant $\bar{\kappa}^{ \pm}$. Since $\bar{\kappa}^{ \pm}= \pm \kappa_{p}-1$, this means that $\kappa_{p}^{ \pm}$is constant $\kappa^{ \pm}$.

We now assume that $\bar{\kappa}^{ \pm} \neq 0$. By the assumption, we have $-\mathbf{e}_{u_{i}}=\kappa \mathbf{X}_{u_{i}}$ (for $i=1, \cdots, n-1$ ), so that there exists a constant vector a such that, $\mathbf{a}=\kappa \mathbf{X}(u)+\mathbf{e}(u)$ for any $u \in U$. If $|\kappa|=\left|\bar{\kappa}^{ \pm}+1\right| \neq 0$, then the vector $\mathbf{v}=(1 / \kappa) \mathbf{a}$ satisfies $\langle\mathbf{v}, \mathbf{v}\rangle=1-1 / \kappa^{2}$ and $\langle\mathbf{X}, \mathbf{v}\rangle=+1$, so that the assertion (a), (b) follows. If $\kappa=0$, then $\mathbf{v}=\mathbf{a}$ satisfies $\langle\mathbf{v}, \mathbf{v}\rangle=-1,\langle\mathbf{X}, \mathbf{v}\rangle=0$. so that the assertion (c) follows.

Finally, we assume that $\bar{\kappa}^{ \pm}=0$. In this case, we have $\mathbb{L}_{u_{i}}^{ \pm}=0$ (for $i=1, \cdots, n-1$ ), so that $\mathbb{L}^{ \pm}$is constant. Therefore we apply Proposition 2.2. This completes the proof.

Let $p=\mathbf{X}\left(u_{0}\right) \in M$ be an umbilic point, we say that $p$ is a positive (or negative) lightcone flat point (or, briefly an $L^{ \pm}$-flat point) if $\bar{\kappa}^{ \pm}=0$. 
Since $\mathbf{X}_{u_{i}}$ (for $i=1, \cdots, n-1$ ) are spacelike vectors, we have the Riemannian metric (the first fundamental form) $d s^{2}=\sum_{i, j=1}^{n-1} g_{i j} d u_{i} d u_{j}$ on $M=\mathbf{X}(U)$, where $g_{i j}(u)=\left\langle\mathbf{X}_{u_{i}}(u), \mathbf{X}_{u_{j}}(u)\right\rangle$ for any $u \in U$. We also define a positive (or negative) lightcone second fundamental form by $\bar{h}_{i j}^{ \pm}(u)=\left\langle-\mathbb{L}_{u_{i}}^{ \pm}(u), \mathbf{X}_{u_{j}}(u)\right\rangle$, and a second fundamental invariant by $h_{i j}(u)=\left\langle-\mathbf{e}_{u_{i}}(u), \mathbf{X}_{u_{j}}(u)\right\rangle$ for any $u \in U$. By definition, we have the following relation:

$$
\bar{h}_{i j}^{ \pm}(u)=-g_{i j}(u) \pm h_{i j}(u) .
$$

The following proposition is analogous to ([5], Proposition 2.4):

Proposition 2.4. Under the above notation, we have the following Weingarten formula:

$$
\mathbb{L}_{u_{i}}^{ \pm}=-\sum_{j=1}^{n-1}\left(\bar{h}^{ \pm}\right)_{i}^{j} \mathbf{X}_{u_{j}},
$$

where $\left(\left(\bar{h}^{ \pm}\right)_{i}^{j}\right)=\left(\bar{h}_{i k}^{ \pm}\right)\left(g^{k j}\right)$ and $\left(g^{k j}\right)=\left(g_{k j}\right)^{-1}$.

As a corollary of the above proposition, we have an explicit expression for the lightcone Gauss-Kronecker curvature by Riemannian metric and the lightcone second fundamental invariant.

Corollary 2.5. With the same notation as in the above proposition, the lightcone GaussKronecker curvature is given by

$$
K_{\ell}^{ \pm}=\operatorname{det}\left(\bar{h}_{i j}^{ \pm}\right) / \operatorname{det}\left(g_{\alpha \beta}\right)
$$

We say that $p=\mathbf{X}\left(u_{0}\right)$ is a positive (or negative) lightcone parabolic point (or, briefly an $L^{ \pm}$-parabolic point) of $\mathbf{X}$ if $K_{\ell}^{ \pm}\left(u_{0}\right)=0$.

\section{$3 \quad$ Lightcone height functions}

In this section we introduce families of functions on a spacelike hypersurface in de Sitter space, which are useful for the study of singularities of lightcone Gauss images and lightcone Gauss maps. Let $\mathbf{X}: U \longrightarrow S_{1}^{n}$ be a hypersurface. We define a family of functions

$$
H: U \times L C^{*} \longrightarrow \mathbb{R}
$$

by $H(u, \mathbf{v})=\langle\mathbf{X}(u), \mathbf{v}\rangle-1$. We call $H$ a lightcone height function on $\mathbf{X}: U \longrightarrow S_{1}^{n}$. we have the following proposition:

Proposition 3.1. Let $H: U \times L C^{*} \longrightarrow \mathbb{R}$ be a lightcone height function on $\mathbf{X}$. Then

(1) $\mathrm{H}(\mathrm{u}, \mathbf{v})=0$ if and only if there exist real numbers $\mu, \xi_{i} \in \mathbb{R}(i=1, \cdots, n-1)$ such that $\mathbf{v}=\mathbf{X}(u)+\mu \mathbf{e}(u)+\xi_{1} \mathbf{X}_{u_{1}}(u)+\cdots+\xi_{n-1} \mathbf{X}_{u_{n-1}}(u)$.

(2) $H(u, \mathbf{v})=\partial H(u, \mathbf{v}) / \partial u_{i}=0($ for $i=1, \cdots, n-1)$ if and only if $\mathbf{v}=\mathbb{L}^{ \pm}(u)$. 
We denote the Hessian matrix of the lightcone height function $h_{\mathbf{v}_{\mathbf{0}}}^{ \pm}(u)=H\left(u, \mathbf{v}_{\mathbf{0}}\right)$ at $u_{0}$ by $\operatorname{Hess}\left(h_{\mathbf{v}_{\mathbf{0}}}^{ \pm}\right)\left(u_{0}\right)$.

Proposition 3.2. Let $\mathbf{X}: U \longrightarrow S_{1}^{n}$ be a hypersurface in $S_{1}^{n}$ and $\mathbf{v}_{\mathbf{0}}^{ \pm}=\mathbb{L}^{ \pm}\left(u_{0}\right)$. Then

(1) $p=\mathbf{X}\left(u_{0}\right)$ is an $L^{ \pm}$-parabolic point if and only if det Hess $\left(h_{\mathbf{v}_{\mathbf{0}}}^{ \pm}\right)\left(u_{0}\right)=0$.

(2) $p=\mathbf{X}\left(u_{0}\right)$ is an $L^{ \pm}$-flat point if and only if rank Hess $\left(h_{\mathbf{v}_{\mathbf{0}}}^{ \pm}\right)\left(u_{0}\right)=0$.

The proofs for the above propositions are parallel to those of Propositions 3.1, 3.2 in [5], so that we omit these.

\section{Lightcone Gauss images as wave fronts}

In this section we naturally interpret the lightcone Gauss image of a spacelike hypersurface in $S_{1}^{n}$ as a wave front set in the theory of Legendrian singularities.

Let $\pi^{ \pm}: P T\left(L C_{ \pm}^{*}\right) \longrightarrow L C_{ \pm}^{*}$ be the projective cotangent bundles with canonical contact structures. Consider the tangent bundle $\tau^{ \pm}: T P T^{*}\left(L C_{ \pm}^{*}\right) \longrightarrow P T^{*}\left(L C_{ \pm}^{*}\right)$ and the differential map $d \pi^{ \pm}: T P T\left(L C_{ \pm}^{*}\right) \longrightarrow T\left(L C_{ \pm}^{*}\right)$ of $\pi^{ \pm}$. For any $X \in T P T^{*}\left(L C_{ \pm}^{*}\right)$, there exists an element $\alpha \in T^{*}\left(L C_{ \pm}^{*}\right)$ such that $\tau^{ \pm}(X)=[\alpha]$. For an element $V \in T_{x}\left(L C_{ \pm}^{*}\right)$, the property $\alpha(V)=0$ does not depend on the choice of representative of the class $[\alpha]$. Thus, we can define the canonical contact structure on $P T^{*}\left(L C_{ \pm}^{*}\right)$ by

$$
K=\left\{X \in T P T^{*}\left(L C_{ \pm}^{*}\right) \mid \tau^{ \pm}(X)\left(d \pi^{ \pm}(X)\right)=0\right\} .
$$

On the other hand, we consider a point $\mathbf{v}=\left(v_{0}, v_{1}, \cdots, v_{n}\right) \in L C_{ \pm}^{*}$, then we have the relation $v_{0}= \pm \sqrt{v_{1}^{2}+\cdots+v_{n}^{2}}$. So we adopt the coordinate system $\left(v_{1}, \cdots, v_{n}\right)$ of the manifold $L C_{ \pm}^{*}$. Then we have the trivialization $P T^{*}\left(L C_{ \pm}^{*}\right) \equiv L C_{ \pm}^{*} \times P \mathbb{R}^{n-1}$, and call $\left(\left(v_{0}, \cdots, v_{n}\right),\left[\xi_{1}: \cdots: \xi_{n}\right]\right)$ homogeneous coordinates of $P T^{*}\left(L C_{ \pm}^{*}\right)$, where $\left[\xi_{1}: \cdots: \xi_{n}\right]$ are the homogeneous coordinates of the dual projective space $P \mathbb{R}^{n-1}$.

It is easy to show that $X_{\bullet} \in K_{\bullet}^{ \pm}$if and only if $\sum_{i=1}^{n} \mu_{i} \xi_{i}=0$, where $\bullet=(x,[\xi])$ and $d \pi_{\bullet}^{ \pm}\left(X_{\bullet}\right)=\sum_{i=1}^{n} \mu_{i} \partial / \partial v_{i} \in T_{\bullet} L C_{ \pm}^{*}$. An immersion $i: L \longrightarrow P T^{*}\left(L C_{ \pm}^{*}\right)$ is said to be a Legendrian immersion if $\operatorname{dim} L=n-1$ and $d i_{q}\left(T_{q} L\right) \subset K_{i(q)}$ for any $q \in L$. The map $\pi \circ i$ is also called the Legendrian map and the image $W(i)=\operatorname{image}(\pi \circ i)$, the wave front of $i$. Moreover, $i$ (or the image of $i$ ) is called the Legendrian lift of $W(i)$.

Let $F:\left(\mathbb{R}^{n-1} \times \mathbb{R}^{k},\left(u_{0}, \mathbf{v}_{0}\right)\right) \longrightarrow(\mathbb{R}, 0)$ be a function germ. We say that $F$ is a Morse family of hypersurfaces if the map germ $\Delta^{*} F:\left(\mathbb{R}^{n-1} \times \mathbb{R}^{k},\left(u_{0}, \mathbf{v}_{0}\right)\right) \longrightarrow\left(\mathbb{R}^{n}, \mathbf{0}\right)$ defined by

$$
\Delta^{*} F=\left(F, \frac{\partial F}{\partial u_{1}}, \cdots, \frac{\partial F}{\partial u_{n-1}}\right)
$$

is non singular.

In this case, we have a smooth $(k-1)$-dimensional smooth submanifold,

$$
\Sigma_{*}(F)=\left\{(u, \mathbf{v}) \in\left(\mathbb{R}^{n-1} \times \mathbb{R}^{k},\left(u_{0}, \mathbf{v}_{0}\right) \mid F(u, \mathbf{v})=\frac{\partial F}{\partial u_{1}}(u, \mathbf{v})=\cdots=\frac{\partial F}{\partial u_{n-1}}(u, \mathbf{v})=0\right\},\right.
$$


and the map germ $\mathcal{L}_{F}:\left(\Sigma_{*}(F),\left(u_{0}, \mathbf{v}_{0}\right)\right) \longrightarrow P T^{*} \mathbb{R}^{k}$ defined by

$$
\mathcal{L}_{F}(u, \mathbf{v})=\left(v,\left[\frac{\partial F}{\partial u_{1}}(u, \mathbf{v}): \cdots: \frac{\partial F}{\partial u_{n-1}}(u, \mathbf{v})\right]\right)
$$

is a Legendrian immersion germ. Then we have the following fundamental theorem of Arnol'd and Zakalyukin [1, 11].

Proposition 4.1. All Legendrian submanifold germs in $P T^{*} \mathbb{R}^{k}$ are constructed by the above method.

We call $F$ a generating family of $\mathcal{L}_{F}\left(\Sigma_{*}(F)\right)$. Therefore the wave front is $W\left(\mathcal{L}_{F}\right)=\left\{\mathbf{v} \in \mathbb{R}^{k} \mid \exists u \in \mathbb{R}^{n-1}\right.$ such that $\left.F(u, \mathbf{v})=\frac{\partial F}{\partial u_{1}}(u, \mathbf{v})=\cdots=\frac{\partial F}{\partial u_{n-1}}(u, \mathbf{v})=0\right\}$.

We call it the discriminant set of $F$.

Proposition 4.2. The lightcone height function $H: U \times L C^{*} \longrightarrow \mathbb{R}$ is a Morse family.

Proof. For any $\mathbf{v}=\left(v_{0}, \cdots, v_{n}\right) \in L C_{ \pm}^{*}$, we have $v_{0} \neq 0$. Without loss of generality, we assume that $v_{0}=\sqrt{v_{1}^{2}+\cdots+v_{n}^{2}} \geq 0$, so that we have

$$
H(u, \mathbf{v})=-x_{0}(u) \sqrt{v_{1}^{2}+\cdots+v_{n}^{2}}+x_{1}(u) v_{1}+\cdots+x_{n}(u) v_{n}-1,
$$

where $\mathbf{X}(u)=\left(x_{0}(u), \cdots, x_{n}(u)\right)$. We have to prove that the mapping $\Delta^{*} H: U \times L C_{ \pm}^{*} \longrightarrow \mathbb{R}^{n}$ is non-singular on $\left(\Delta^{*} H\right)^{-1}(0)$. But this computation is similar to [5] (see Proposition 4.2), so that we omit it.

Since $H$ is a Morse family of hypersurfaces, we apply the previous arguments. So that we have the Legendrian immersion germ $\mathcal{L}^{ \pm}:\left(\Sigma_{*}^{ \pm}(H),\left(u_{0}, \mathbf{v}_{0}^{ \pm}\right)\right) \longrightarrow P T^{*}\left(L C_{ \pm}^{*}\right)$ by

$$
\mathcal{L}^{ \pm}(u)=\left(\mathbf{v}^{ \pm},\left[\frac{\partial H}{\partial v_{1}}\left(u, \mathbf{v}^{ \pm}\right): \cdots: \frac{\partial H}{\partial v_{n}}\left(u, \mathbf{v}^{ \pm}\right)\right]\right)
$$

where $\mathbf{v}^{ \pm}=\mathbb{L}^{ \pm}(u)$ and $\Sigma_{*}^{ \pm}(H)$ is a singular set of $H$

$$
\Sigma_{*}^{ \pm}(H)=\left(\Delta^{*} H\right)^{-1}(0)=\left\{(u, \mathbf{v}) \in U \times L C_{ \pm}^{*} \mid \mathbf{v}=\mathbb{L}^{ \pm}(u)\right\} .
$$

Therefore, we have the Legendrian immersion $\mathcal{L}^{ \pm}$whose wave front set is the lightcone Gauss image $\mathbb{L}^{ \pm}$. 


\section{Contact with de Sitter hyperhorospheres}

In this section we start to review the theory of contact due to Montaldi [7]. Let $X_{i}$ and $Y_{i}$ (for $i=1,2)$ be submanifolds of $\mathbb{R}^{n}$ with $\operatorname{dim} X_{1}=\operatorname{dim} X_{2}$ and $\operatorname{dim} Y_{1}=\operatorname{dim} Y_{2}$. We say that the contact of $X_{1}$ and $Y_{1}$ at $y_{1}$ is the same type as the contact of $X_{2}$ and $Y_{2}$ at $y_{2}$ if there is a diffeomorphism germ $\Phi:\left(\mathbb{R}^{n}, y_{1}\right) \longrightarrow\left(\mathbb{R}^{n}, y_{2}\right)$ such that $\Phi\left(X_{1}\right)=X_{2}$ and $\Phi\left(Y_{1}\right)=Y_{2}$. In this case we write $K\left(X_{1}, Y_{1} ; y_{1}\right)=K\left(X_{2}, Y_{2} ; y_{2}\right)$.

Two function germs $g_{1}, g_{2}:\left(\mathbb{R}^{n}, a_{i}\right) \longrightarrow(\mathbb{R}, 0)(i=1,2)$ are $\mathcal{K}$-equivalent if there are a diffeomorphism germ $\Phi:\left(\mathbb{R}^{n}, \mathbf{a}_{\mathbf{1}}\right) \longrightarrow\left(\mathbb{R}^{n}, \mathbf{a}_{\mathbf{2}}\right)$, and a function germ $\lambda:\left(\mathbb{R}^{n}, \mathbf{a}_{\mathbf{1}}\right) \longrightarrow \mathbb{R}$ with $\lambda\left(\mathbf{a}_{\mathbf{1}}\right) \neq 0$ such that $f_{1}=\lambda \cdot\left(g_{2} \circ \Phi\right)$. In [7] Montaldi has shown the following theorem.

Theorem 5.1. (Montaldi [7]) Let $X_{i}$ and $Y_{i}$ (for $i=1,2$ ) be submanifolds of $\mathbb{R}^{n}$ with $\operatorname{dim} X_{1}=$ $\operatorname{dim} X_{2}$ and $\operatorname{dim} Y_{1}=\operatorname{dim} Y_{2}$. Let $g_{i}:\left(X_{1}, x_{1}\right) \longrightarrow\left(\mathbb{R}^{n}, y_{i}\right)$ be immersion germs and $f_{i}$ : $\left(\mathbb{R}^{n}, y_{i}\right) \longrightarrow\left(\mathbb{R}^{p}, \mathbf{0}\right)$ be submersion germs with $\left(Y_{i}, y_{i}\right)=\left(f_{i}^{-1}(\mathbf{0}), y_{i}\right)$. Then $K\left(X_{1}, Y_{1} ; y_{1}\right)=$ $K\left(X_{2}, Y_{2} ; y_{2}\right)$. if and only if $f_{1} \circ g_{1}$ and $f_{2} \circ g_{2}$ are $\mathcal{K}$-equivalent.

We can apply the above theorem to our case. Let $\mathbf{v}_{\mathbf{0}} \in L C^{*}$. We define $\mathfrak{h}_{\mathbf{v}_{\mathbf{0}}}: S_{1}^{n} \longrightarrow \mathbb{R}$ by $\mathfrak{h}_{\mathbf{v}_{\mathbf{0}}}(w)=\left\langle w, \mathbf{v}_{\mathbf{0}}\right\rangle-1$. Then we have a de Sitter hyperhorosphere $\mathfrak{h}_{\mathbf{v}_{\mathbf{0}}}^{-1}(0)=H P\left(\mathbf{v}_{\mathbf{0}},+1\right) \cap S_{1}^{n}$. We write $H S\left(\mathbf{v}_{\mathbf{0}},+1\right)=H P\left(\mathbf{v}_{\mathbf{0}},+1\right) \cap S_{1}^{n}$. For any $u_{0} \in U$, we consider the lightlike vector $\mathbf{v}_{\mathbf{0}}{ }^{ \pm}=\mathbb{L}^{ \pm}\left(u_{0}\right)$. Then we have

$$
\mathfrak{h}_{\mathbf{v}_{\mathbf{0}}^{ \pm}} \circ \mathbf{X}\left(u_{0}\right)=H\left(u_{0}, \mathbb{L}^{ \pm}\left(u_{0}\right)\right)=0 .
$$

We also have relations

$$
\frac{\partial \mathfrak{h}_{\mathbf{v}_{0}^{ \pm}} \circ \mathbf{X}}{\partial u_{i}}\left(u_{0}\right)=\left\langle\mathbf{X}_{u_{i}}\left(u_{0}\right), \mathbb{L}^{ \pm}\left(u_{0}\right)\right\rangle=0
$$

for $i=1, \cdots, n-1$. This means that the de Sitter hyperhorosphere $\mathfrak{h}_{\mathbf{v}_{\mathbf{0}}^{ \pm}}^{-1}(0)=H S\left(\mathbf{v}_{\mathbf{0}}^{ \pm},+1\right)$ is tangent to $M=\mathbf{X}(U)$ at $p=\mathbf{X}\left(u_{0}\right)$. In this case, we call $H S\left(\mathbf{v}_{\mathbf{0}}^{ \pm},+1\right)$ the tangent de Sitter hyperhorosphere of $M=\mathbf{X}$ at $p=\mathbf{X}(u)$ (or $u_{0}$ ), which we write $H S^{ \pm}\left(\mathbf{X}, u_{0}\right)$. Let $\mathbf{v}_{\mathbf{1}}$ and $\mathbf{v}_{\mathbf{2}}$ be lightlike vectors. We say that $H S\left(\mathbf{v}_{\mathbf{1}},+1\right)$ and $H S\left(\mathbf{v}_{\mathbf{2}},+1\right)$ are parallel if $\mathbf{v}_{\mathbf{1}}$ and $\mathbf{v}_{\mathbf{2}}$ are linearly independent. Then we have the following proposition.

Proposition 5.2. Let $\mathbf{X}: U \longrightarrow S_{1}^{n}$ be a hypersurface. Consider two points $u_{1}, u_{2} \in U$. Then $\mathbb{L}^{ \pm}\left(u_{1}\right)=\mathbb{L}^{ \pm}\left(u_{2}\right)$ if and only if $H S^{ \pm}\left(\mathbf{X}, u_{1}\right)=H S^{ \pm}\left(\mathbf{X}, u_{2}\right)$.

We now review some notions of Legendrian singularity theory to study the contact between hypersurfaces and de Sitter hyperhorospheres. We say that Legendrian immersion germs $i_{j}:\left(U_{j}, u_{j}\right) \longrightarrow\left(P T^{*} \mathbb{R}^{n}, p_{j}\right)(j=1,2)$ are Legendrian equivalent if there exists a contact diffeomorphism germ $H:\left(P T^{*} \mathbb{R}^{n}, p_{1}\right) \longrightarrow\left(P T^{*} \mathbb{R}^{n}, p_{2}\right)$ such that $H$ preserves fibers of $\pi$ and $H\left(U_{1}\right)=U_{2}$. A Legendrian immersion germ $i_{1}$ is said Legendrian stable if there are a neighborhood $U$ in the map space of Legendrian immersions (in the Whitney $C^{\infty}$ topology) such that for any elements of $U$ are Legendrian equivalent to $i_{1}$. 
Proposition 5.3. (Zakalyukin [12]) Let $i_{1}, i_{2}$ be Legendrian immersion germs such that regular sets of $\pi \circ i_{1}$ and $\pi \circ i_{2}$ are respectively dense. Then $i_{1}, i_{2}$ are Legendrian equivalent if and only if corresponding wave front sets $W\left(i_{1}\right)$ and $W\left(i_{2}\right)$ are diffeomorphic as set germs.

Let $F_{i}:\left(\mathbb{R}^{n} \times \mathbb{R}^{k},\left(a_{i}, b_{i}\right)\right) \longrightarrow(\mathbb{R}, c)(k=1,2)$ be $k$-parameter unfoldings of function germs $f_{i}$, we say $F_{1}$ and $F_{2}$ are $\mathcal{P}$ - $\mathcal{K}$-equivalent if there exists a diffeomorphism germ $\Phi$ : $\left(\mathbb{R}^{n} \times \mathbb{R}^{k},\left(a_{1}, b_{1}\right)\right) \longrightarrow\left(\mathbb{R}^{n} \times \mathbb{R}^{k},\left(a_{2}, b_{2}\right)\right)$ of the form $\Phi(u, x)=\left(\phi_{1}(u, x), \phi_{2}(x)\right)$ for $(u, x) \in$ $\mathbb{R}^{n} \times \mathbb{R}^{k}$ and a function germ $\lambda:\left(\mathbb{R}^{n} \times \mathbb{R}^{k},\left(a_{1}, b_{1}\right)\right) \longrightarrow \mathbb{R}$ such that $\lambda\left(a_{1}, b_{1}\right) \neq 0$ and $F_{1}(u, x)=\lambda(u, x) \cdot\left(F_{2} \circ \Phi\right)(u, x)$.

Theorem 5.4. (Arnol'd, Zakalyukin $[1,11])$ Let $F, G:\left(\mathbb{R}^{k} \times \mathbb{R}^{n}, \mathbf{0}\right) \longrightarrow(\mathbb{R}, \mathbf{0})$ be Morse families and denote their Legendrian immersion by $\mathcal{L}_{F}, \mathcal{L}_{G}$. Then

(1) $\mathcal{L}_{F}$ and $\mathcal{L}_{G}$ are Legendrian equivalent if and only if $F$ and $G$ are $\mathcal{P}$-K $\mathcal{K}$-equivalent.

(2) $\mathcal{L}_{F}$ is Legendrian stable if and only if $F$ is $\mathcal{K}$-versal deformation.

Let $\mathbb{L}_{i}^{ \pm}:\left(U, u_{i}\right) \longrightarrow\left(L C_{ \pm}^{*}, \mathbf{v}_{\mathbf{i}}^{ \pm}\right)$(for $\left.i=1,2\right)$ be de Sitter Gauss image germs of hypersurface germs $\mathbf{X}_{i}:\left(U, u_{i}\right) \longrightarrow\left(S_{1}^{n}, p_{i}\right)$. We say $\mathbb{L}_{1}^{ \pm}$and $\mathbb{L}_{2}^{ \pm}$are $\mathcal{A}$-equivalent if and only if there exist diffeomorphism germs $\phi:\left(U, u_{1}\right) \longrightarrow\left(U, u_{2}\right)$ and $\Phi:\left(S_{1}^{n}, \mathbf{v}_{1}^{ \pm}\right) \longrightarrow\left(S_{1}^{n}, \mathbf{v}_{2}^{ \pm}\right)$such that $\Phi \circ \mathbb{L}_{1}^{ \pm}=\mathbb{L}_{2}^{ \pm} \circ \phi$.

We denote $h_{i, \mathbf{v}_{\mathbf{i}}^{ \pm}}:\left(U, u_{i}\right) \longrightarrow(\mathbb{R}, \mathbf{0})$ by $h_{i, \mathbf{v}_{\mathbf{i}}^{ \pm}}(u)=H_{i}\left(u, \mathbf{v}_{\mathbf{i}}^{ \pm}\right)$; Then we have $h_{i, \mathbf{v}_{\mathbf{i}}^{ \pm}}(u)=$ $\left(\mathfrak{h}_{\mathbf{v}_{\mathbf{i}}^{ \pm}}\right) \circ \mathbf{X}_{i}(u)$. By Theorem 5.1,

$$
K\left(\mathbf{X}_{\mathbf{1}}(U), H S^{ \pm}\left(\mathbf{X}_{\mathbf{1}}, u_{1}\right), \mathbf{v}_{1}^{ \pm}\right)=K\left(\mathbf{X}_{\mathbf{2}}(U), H S^{ \pm}\left(\mathbf{X}_{\mathbf{2}}, u_{2}\right), \mathbf{v}_{2}^{ \pm}\right)
$$

if and only if $h_{1, \mathbf{v}_{1}^{ \pm}}$and $h_{2, \mathbf{v}_{2}^{ \pm}}$are $\mathcal{K}$-equivalent.

We denote $Q^{ \pm}\left(\mathbf{X}, u_{0}\right)$ the local ring of the function germ $h_{\mathbf{v}_{0}^{ \pm}}:\left(U, u_{0}\right) \longrightarrow \mathbb{R}$ by

$$
Q^{ \pm}\left(\mathbf{X}, u_{0}\right)=C_{u_{0}}^{\infty}(U) /\left\langle h_{\mathbf{v}_{0}}\right\rangle_{C_{u_{0}}^{\infty}}
$$

where $\mathbf{v}_{0}^{ \pm}=\mathbb{L}^{ \pm}\left(u_{0}\right)$ and $C_{u_{0}}^{\infty}(U)$ is the local ring of function germs at $u_{0}$ with the unique maximal ideal $\mathfrak{M}$.

Proposition 5.5. Let $F, G:\left(\mathbb{R}^{k} \times \mathbb{R}^{n}, \mathbf{0}\right) \longrightarrow(\mathbb{R}, \mathbf{0})$ be Morse families. Suppose that Legendrian immersion germs $\mathcal{L}_{F}$ and $\mathcal{L}_{G}$ are Legendrian stable, then the following conditions are equivalent:

(1) $\left(W\left(\mathcal{L}_{F}\right), \mathbf{0}\right)$ and $\left(W\left(\mathcal{L}_{G}\right), \mathbf{0}\right)$ are diffeomorphic as set germs.

(2) $\mathcal{L}_{F}$ and $\mathcal{L}_{G}$ are Legendrian equivalent.

(3) $Q(f)$ and $Q(g)$ are isomorphic as $\mathbb{R}$-algebras, where $f=\left.F\right|_{\mathbb{R}^{k} \times\{\mathbf{0}\}}$ and $g=\left.G\right|_{\mathbb{R}^{k} \times\{\mathbf{0}\}}$.

By the above propositions, we have following theorem. 
Theorem 5.6. Let $\mathbf{X}_{i}:\left(U, u_{i}\right) \longrightarrow\left(S_{1}^{n}, p_{i}\right)$ (for $\left.i=1,2\right)$ be hypersurface germs such that the corresponding Legendrian immersion germs are Legendrian stable. Then the following conditions are equivalent:

(1) Lightcone Gauss image germs $\mathbb{L}_{1}^{ \pm}$and $\mathbb{L}_{2}^{ \pm}$are $\mathcal{A}$-equivalent.

(2) Legendrian immersion germs $\mathcal{L}_{1}$ and $\mathcal{L}_{2}$ are Legendrian equivalent.

(3) Lightcone height function germs $H_{1}$ and $H_{2}$ are $\mathcal{P}$ - $\mathcal{K}$-equivalent.

(4) $h_{1, \mathbf{v}_{1}^{ \pm}}$and $h_{2, \mathbf{v}_{2}^{ \pm}}$are $\mathcal{K}$-equivalent.

(5) $K\left(\mathbf{X}_{1}(U), H S^{ \pm}\left(\mathbf{X}_{1}, u_{1}\right), \mathbf{v}_{1}^{ \pm}\right)=K\left(\mathbf{X}_{2}(U), H S^{ \pm}\left(\mathbf{X}_{2}, u_{2}\right), \mathbf{v}_{2}^{ \pm}\right)$

(6) Local rings $Q^{ \pm}\left(\mathbf{X}_{1}, u_{1}\right)$ and $Q^{ \pm}\left(\mathbf{X}_{2}, u_{2}\right)$ are isomorphic as $\mathbb{R}$-algebras.

Proof. Since $\mathcal{L}_{1}$ and $\mathcal{L}_{2}$ are Legendrian stable, regular sets of $\mathbb{L}_{1}$ and $\mathbb{L}_{2}$ are respectively dense, by Proposition 5.3, the conditions (1) and (2) are equivalent. And we apply Theorem 5.4, the conditions (2) and (3) are equivalent. By the previous arguments from Theorem 5.1, the conditions (4) and (5) are equivalent.

If we assume the condition $(3)$, then $\mathcal{P}$ - $\mathcal{K}$-equivalence preserves the $\mathcal{K}$-equivalence, so that the condition (4) holds. Since the local ring $Q^{ \pm}\left(\mathbf{X}_{i}, u_{i}\right)$ is $\mathcal{K}$-invariant, this means that the condition (6) holds. By Proposition 5.5, the condition (6) implies the condition (2).

In the next section, we will prove that the assumption of the Theorem 5.6 is generic property in the case when $n \leq 6$. In general we have the following proposition.

Proposition 5.7. Let $\mathbf{X}_{i}:\left(U, u_{i}\right) \longrightarrow\left(S_{1}^{n}, p_{i}\right)$ (for $\left.i=1,2\right)$ be hypersurface germs such that their $L^{ \pm}$-parabolic sets have no interior points as subspaces of $U$. If lightcone Gauss image germs $\mathbb{L}_{1}^{ \pm}$and $\mathbb{L}_{2}^{ \pm}$are $\mathcal{A}$-equivalent, then

$$
K\left(\mathbf{X}_{1}(U), H S^{ \pm}\left(\mathbf{X}_{1}, u_{1}\right), \mathbf{v}_{1}^{ \pm}\right)=K\left(\mathbf{X}_{2}(U), H S^{ \pm}\left(\mathbf{X}_{2}, u_{2}\right), \mathbf{v}_{2}^{ \pm}\right) .
$$

In this case, $\left(\mathbf{X}_{1}^{-1}\left(H S\left(\mathbb{L}_{1}^{ \pm}\left(u_{1}\right),+1\right)\right), u_{1}\right)$ and $\left(\mathbf{X}_{2}^{-1}\left(H S\left(\mathbb{L}_{2}^{ \pm}\left(u_{2}\right),+1\right)\right), u_{2}\right)$ are diffeomorphic as set germs.

Proof. Since the $L^{ \pm}$-parabolic point set is a singular set of the lightcone Gauss image, the corresponding Legendrian immersion germs $\mathcal{L}_{i}$ satisfy the hypothesis of Proposition 5.3. If $\mathbb{L}_{1}^{ \pm}$ and $\mathbb{L}_{2}^{ \pm}$are $\mathcal{A}$-equivalent, then $\mathcal{L}_{1}$ and $\mathcal{L}_{2}$ are Legendrian equivalent. By Theorem 5.4, $H_{1}$ and $H_{2}$ are $\mathcal{P}$ - $\mathcal{K}$-equivalent, so that $h_{1, \mathbf{v}_{1}^{ \pm}}$and $H_{2, \mathbf{v}_{2}^{ \pm}}$are $\mathcal{K}$-equivalent. Applying Theorem 5.1, the first assertion holds.

On the other hand, we have $\left(\mathbf{X}_{i}^{-1}\left(H S\left(\mathbb{L}_{i}^{ \pm}\left(u_{i}\right),+1\right)\right), u_{i}\right)=\left(h_{i, \mathbf{v}_{i}^{ \pm}}^{-1}(0), u_{i}\right)$. Since $\mathcal{K}$-equivalence preserves the zero level sets, $\left(\mathbf{X}_{1}^{-1}\left(H S\left(\mathbb{L}_{1}^{ \pm}\left(u_{1}\right),+1\right)\right), u_{1}\right)$ and $\left(\mathbf{X}_{2}^{-1}\left(H S\left(\mathbb{L}_{2}^{ \pm}\left(u_{2}\right),+1\right)\right), u_{2}\right)$ are diffeomorphic as set germs. 
For a hypersurface germ $\mathbf{X}$, we call $\left(\mathbf{X}^{-1}\left(H S\left(\mathbb{L}^{ \pm}\left(u_{0}\right),+1\right)\right), u_{0}\right)$ the tangent de Sitter hyperhorospherical indicatrix germ of $\mathbf{X}$. By Proposition 5.7, the diffeomorphic type of the tangent de Sitter horospherical indicatrix germ is an invariant under the $\mathcal{A}$-equivalence among lightcone Gauss image germs. We define the $\mathcal{K}$-codimension (or Tyurina number) of the function germ $h_{\mathbf{v}_{0}^{ \pm}}$by

$$
\operatorname{H-ord}^{ \pm}\left(\mathbf{X}, u_{0}\right)=\operatorname{dim} C_{u_{0}}^{\infty} /\left\langle h_{\mathbf{v}_{0}^{ \pm}}\left(u_{0}\right), \partial h_{\mathbf{v}_{0}^{ \pm}}\left(u_{0}\right) / \partial u_{i}\right\rangle_{C_{u_{0}}^{\infty}},
$$

where $\mathbf{v}_{0}^{ \pm}=\mathbb{L}^{ \pm}\left(u_{0}\right)$. We also have the notion of corank of the function germ:

$$
\mathrm{H}-\operatorname{corank}^{ \pm}\left(\mathbf{X}, u_{0}\right)=(n-1)-\operatorname{rank} \operatorname{Hess}\left(h_{\mathbf{v}_{0}^{ \pm}}\left(u_{0}\right)\right) \text {. }
$$

By Proposition 3.2, $p=\mathbf{X}\left(u_{0}\right)$ is a $L^{ \pm}$-parabolic point if and only if $\mathrm{H}$-corank ${ }^{ \pm}\left(\mathbf{X}, u_{0}\right) \geq 1$. Moreover $p$ is a $L^{ \pm}$-flat point if and only if $\mathrm{H}-\operatorname{ord}^{ \pm}\left(\mathbf{X}, u_{0}\right)=n-1$.

We say a function germ $f:\left(\mathbb{R}^{n-1}, \mathbf{a}\right) \longrightarrow \mathbb{R}$ has the $\mathcal{A}_{k}$-type singularity at a if $f$ is $\mathcal{K}$ equivalent to the germ

$$
g\left(u_{1}, \cdots, u_{n-1}\right)= \pm u_{1} \pm \cdots \pm u_{n-2}+u_{n-1}^{k+1} .
$$

\section{$6 \quad$ Generic properties}

In this section we consider generic properties of hypersurfaces in $S_{1}^{n}$. We consider the map space of spacelike embeddings $\operatorname{Sp}-\operatorname{Emb}\left(U, S_{1}^{n}\right)$ with Whitney $C^{\infty}$-topology. We define the function $\mathcal{H}: S_{1}^{n} \times L C^{*} \longrightarrow \mathbb{R}$ by

$$
\mathcal{H}(\mathbf{x}, \mathbf{v})=\langle\mathbf{x}, \mathbf{v}\rangle-1
$$

and denote $\mathcal{H}_{\mathbf{x}}(\mathbf{v})=\mathcal{H}(\mathbf{x}, \mathbf{v})$. Then $\mathcal{H}_{\mathbf{x}}$ is a submersion for any $\mathbf{x} \in S_{1}^{n}$. For any spacelike hypersurface $\mathbf{X} \in \operatorname{Sp}-\operatorname{Emb}\left(U, S_{1}^{n}\right)$, we have $H=\mathcal{H} \circ\left(\mathbf{X} \times \operatorname{id}_{L C^{*}}\right)$. We also have the $\ell$-jet extension

$$
j_{1}^{\ell} \mathcal{H}: U \times L C^{*} \longrightarrow J^{\ell}(U, \mathbb{R})
$$

defined by $j_{1}^{\ell} \mathcal{H}(\mathbf{x}, \mathbf{v})=j^{\ell} h_{\mathbf{v}}(u)$. We consider the trivialization

$$
J^{\ell}(U, \mathbb{R}) \equiv U \times \mathbb{R} \times J^{\ell}(n-1,1) .
$$

For any submanifold $Q \subset J^{\ell}(n-1,1)$, we denote $\widetilde{Q}=U \times\{0\} \times Q$. Then we have the following proposition as a corollary of Lemma 6 of Wassermann.

Proposition 6.1. (Wassermann [10]) Let $Q$ be a submanifold of $J^{\ell}(n-1,1)$. Then the set

$$
T_{Q}=\left\{\mathbf{X} \in \operatorname{Sp}-\operatorname{Emb}\left(U, S_{1}^{n}\right) \mid j_{1}^{\ell} H \text { is transversal to } \widetilde{Q}\right\}
$$

is a residual subset of $\operatorname{Sp}-\operatorname{Emb}\left(U, S_{1}^{n}\right)$. If $Q$ is a closed subset, then $T_{Q}$ is open. 
We remark that if corresponding height function $h_{\mathbf{v}_{0}}$ is $\ell$-determined relative to $\mathcal{K}$, then $H$ is a $\mathcal{K}$-versal deformation if and only if $H$ is transversal to $\widetilde{\mathcal{K}}_{h_{v_{0}}}^{\ell}$, where $\mathcal{K}_{h_{v_{0}}}^{\ell}$ is the $\mathcal{K}$-orbit through $j^{\ell} h_{\mathbf{v}_{0}}(\mathbf{0}) \in J^{\ell}(n-1,1)$. Applying Theorem 5.4, this condition is equivalent to the condition that corresponding Legendrian immersion germ is Legendrian stable. From the previous arguments and the appendix of [5], we have following proposition. (See also [1].)

Theorem 6.2. if $n \leq 6$, there exists an open subset $\mathcal{O} \subset \operatorname{Sp}-\operatorname{Emb}\left(U, S_{1}^{n}\right)$ such that for any $\mathbf{X} \in \mathcal{O}$, corresponding Legendrian immersion germ $\mathcal{L}$ is Legendrian stable.

\section{Spacelike de Sitter Monge form}

In this section we consider the analogous notion for a spacelike hypersurface in de Sitter $n$ space. We now consider the function $f\left(u_{1}, \cdots, u_{n-1}\right)$ with $f(0)=0$ and $f_{u_{i}}(0)=0$. Then we have a spacelike hypersurface in $S_{1}^{n}$ by

$$
\mathbf{X}_{f}(u)=\left(-f(u),-\sqrt{1+f^{2}(u)-u_{1}^{2}-\cdots-u_{n-1}^{2}}, u_{1}, \cdots, u_{n-1}\right)
$$

where $U$ is an open neighborhood at $\mathbf{0}$. We can easily calculate $\mathbf{e}(\mathbf{0})=(1,0, \cdots, 0)$; therefore $\mathbb{L}^{ \pm}(\mathbf{0})=( \pm 1,-1,0, \cdots, 0)$. We call $\mathbf{X}_{f}$ a spacelike de Sitter Monge form (briefly, spacelike Monge form). Then we have the following proposition.

Proposition 7.1. Any spacelike surface in $S_{1}^{n}$ is locally given by the spacelike Monge form.

Proof. Let $\mathbf{X}: U \longrightarrow S_{1}^{n}$ be a spacelike hypersurface. Since we can apply Lorentzian motions of Minkowski $(n+1)$-space such that $S_{1}^{n}$ is the invariant set, without loss of generality, we assume that $p=\mathbf{X}(\mathbf{0})=(0,-1,0, \cdots, 0)$. We have a basis $\left\{\mathbf{X}(\mathbf{0}), \mathbf{e}(\mathbf{0}), \mathbf{X}_{u_{1}}(\mathbf{0}), \cdots, \mathbf{X}_{u_{n-1}}(\mathbf{0})\right\}$ of $T_{p} \mathbb{R}^{n+1}$ such that $T_{p} M=\left\langle\mathbf{X}_{u_{1}}(\mathbf{0}), \cdots, \mathbf{X}_{u_{n-1}}(\mathbf{0})\right\rangle_{\mathbb{R}}$. Applying the Gram-Schmidt procedure, we have a pseudo orthonormal basis $\left\{\mathbf{X}(\mathbf{0}), \mathbf{e}(\mathbf{0}), \mathbf{e}_{1}, \cdots, \mathbf{e}_{n-1}\right\}$ of $\mathbb{R}_{1}^{n+1}$ such that $T_{p} M=\left\langle\mathbf{e}_{1}, \cdots, \mathbf{e}_{n-1}\right\rangle_{\mathbb{R}}$.

In particular, $\left\{\mathbf{e}_{1}, \cdots, \mathbf{e}_{n-1}\right\}$ is an orthonormal basis of spacelike subspace $T_{p} M, T_{p} M$ is considered to be a subspace of $\mathbb{R}_{0}^{n}=\left\{\left(0, x_{1}, \cdots, x_{n}\right) \mid x_{i} \in \mathbb{R}\right\}$. By a rotation of the space $\mathbb{R}_{0}^{n}$, we assume that $T_{p} M=\left\{\left(0,0, x_{2}, \cdots, x_{n}\right) \mid x_{i} \in \mathbb{R}\right\}$. We remark that this rotation can be considered to be a Lorentzian motion of $\mathbb{R}_{1}^{n+1}$.

Therefore, the hypersurface germ $(M, p)$ is written in the form

$$
\mathbf{X}(u)=\left(-f(u),-g(u), u_{1}, \cdots, u_{n-1}\right) .
$$

with function germs $f(u), g(u)$. Since $M \subset S_{1}^{n}$, we have a relation $g^{2}(u)=1+f^{2}(u)-u_{1}^{2}-$ $\cdots-u_{n-1}^{2}$. By a rotation of the space $\mathbb{R}_{0}^{n}$, we can assume $g(u) \geq 0$, so that we have

$$
g(u)=\sqrt{1+f^{2}(u)-u_{1}^{2}-\cdots-u_{n-1}^{2}} .
$$

Since $T_{p} M=\left\{\left(0,0, x_{2}, \cdots, x_{n}\right) \mid x_{i} \in \mathbb{R}\right\}$, the conditions $f(\mathbf{0})=f_{u_{i}}(\mathbf{0})=0(i=1, \cdots, n-1)$ are automatically satisfied. This completes the proof. 
For the lightlike vector $\mathbf{v}_{\mathbf{0}}=( \pm 1,-1,0, \cdots, 0)$, we consider the de Sitter hyperhorosphere $H S\left(\mathbf{v}_{\mathbf{0}},+1\right)$. Then we have the spacelike Monge form of $H S\left(\mathbf{v}_{\mathbf{0}},+1\right)$.

$$
\mathbf{X}_{H S^{ \pm}}=\left(\mp \frac{1}{2}\left(u_{1}^{2}+\cdots+u_{n-1}^{2}\right),-1+\frac{1}{2}\left(u_{1}^{2}+\cdots+u_{n-1}^{2}\right), u_{1}, \cdots, u_{n-1}\right) .
$$

Here we can check the relation $\left\langle\mathbf{v}_{0}^{ \pm}, \mathbf{X}_{H S^{ \pm}}(u)\right\rangle=1$. On the other hand, we have $\mathbf{X}_{H S^{ \pm}}(\mathbf{0})=$ $(0,-1,0, \cdots, 0)$ and $\mathbf{X}_{H S^{ \pm}, u_{i}}(\mathbf{0})$ is the $x_{i+1^{-}}$-axis for $i=1, \cdots, n-1$. This means that $T_{p} M=$ $T_{p}\left(\mathbf{X}_{H S^{ \pm}}(U)\right)$. Therefore $\mathbf{X}_{H S^{ \pm}}(U) \subset H S\left(\mathbf{v}_{0}^{ \pm},+1\right)$ is the tangent de Sitter hyperhorosphere of $M=\mathbf{X}(U)$ at $p=\mathbf{X}(\mathbf{0})$. It follows from this fact that the tangent de Sitter hyperhorospherical indicatrix germ of the spacelike Monge form $\mathbf{X}_{f}$ is given as follows:

$$
\mathbf{X}_{f}^{-1}\left(H S\left(\mathbf{v}_{0}^{ \pm},+1\right)\right)=\left\{\left(u_{1}, \cdots, u_{n-1}\right) \mid \pm 2 f(u)=u_{1}^{2}+\cdots+u_{n-1}^{2}\right\} .
$$

Since the lightcone height function of $\mathbf{X}_{f}$ at $\mathbf{v}_{0}^{ \pm}$is

$$
h_{\mathbf{v}_{0}^{ \pm}}(u)= \pm f(u)+\sqrt{1+f^{2}(u)-u_{1}^{2}-\cdots-u_{n-1}^{2}}-1
$$

we can calculate the Hessian matrix; then we have Hess $h_{\mathbf{v}_{0}^{ \pm}}(\mathbf{0})= \pm \operatorname{Hess}(f(\mathbf{0}))-\mathrm{I}_{n-1}$, where $\mathrm{I}_{n-1}$ is an identity matrix.

On the other hand, since $f(\mathbf{0})=f_{u_{i}}(\mathbf{0})=0$, We may write

$$
f(u)=\frac{1}{2} \kappa_{1} u_{1}^{2}+\cdots \frac{1}{2} \kappa_{n-1} u_{n-1}^{2}+g(u)
$$

where $g \in \mathfrak{M}_{n-1}^{3}$ and $\kappa_{1}, \cdots, \kappa_{n-1}$ are eigenvalues of Hess $(f(\mathbf{0}))$. Under this representation, we can easily calculate $\left(\mathbf{X}_{f}\right)_{u_{i}, u_{j}}(\mathbf{0})=\left(-f_{u_{i}, u_{j}}(\mathbf{0}), \delta_{i j}, 0, \cdots, 0\right)$. It follows from that this fact

$$
\bar{h}_{i j}^{ \pm}(\mathbf{0})= \pm f_{u_{i}, u_{j}}(\mathbf{0})-\delta_{i j}=\delta_{i j}\left( \pm \kappa_{i}-1\right)
$$

and $g_{i j}(\mathbf{0})=\delta_{i j}$. Therefore, we have $\bar{\kappa}_{i}^{ \pm}(\mathbf{0})=-1 \pm \kappa_{i}$ and

$$
K_{\ell}^{ \pm}(\mathbf{0})=\prod_{i=1}^{n-1} \bar{\kappa}_{i}^{ \pm}(\mathbf{0})=\prod_{i=1}^{n-1}\left(-1 \pm \kappa_{i}\right)
$$

The tangent de Sitter hyperhorospherical indicatrix germ is given by

$$
\mathbf{X}_{f}^{-1}\left(H S\left(\mathbf{v}_{0}^{ \pm},+1\right)\right)=\left\{\left(u_{1}, \cdots, u_{n-1}\right) \mid \bar{\kappa}_{1}^{ \pm}(\mathbf{0}) u_{1}^{2}+\cdots+\bar{\kappa}_{n-1}^{ \pm}(\mathbf{0}) u_{n-1}^{2} \pm 2 g(u)=0\right\} .
$$

\section{Spacelike surfaces in de Sitter 3-space}

In this section we consider $n=3$. In this case we call $\mathbf{X}: U \longrightarrow S_{1}^{3}$ a spacelike surface and $H S\left(\mathbf{v}_{0},+1\right)$ a de Sitter horosphere etc. By Theorem 6.2 and the classification of function germs [1], we have he following theorem. 
Theorem 8.1. There exists an open dense subset $\mathcal{O} \subset \operatorname{Sp}-\operatorname{Emb}\left(U, S_{1}^{n}\right)$ such that for any $\mathbf{X} \in \mathcal{O}$, the following conditions holds.

(1) The $L^{ \pm}$-parabolic set $K_{\ell}^{-1}(0)$ is a regular curve. We call such a curve the $L^{ \pm}$-parabolic curve.

(2) The lightcone Gauss image $\mathbb{L}^{ \pm}$along the $L^{ \pm}$-parabolic curve is a cuspidal edge except at isolated points. At this points $\mathbb{L}^{ \pm}$is swallowtail.

Here, a map germ $L:\left(\mathbb{R}^{2}, \mathbf{a}\right) \longrightarrow\left(\mathbb{R}^{3}, \mathbf{b}\right)$ is called the cuspidal edge if it is $\mathcal{A}$-equivalent to the germ $\left(u_{1}, u_{2}^{2}, u_{2}^{3}\right)$ and the swallowtail if it is $\mathcal{A}$-equivalent to the germ $\left(3 u_{1}^{4}+u_{1}^{2} u_{2}, 4 u_{1}^{3}+2 u_{1} u_{2}, u_{2}\right)$.

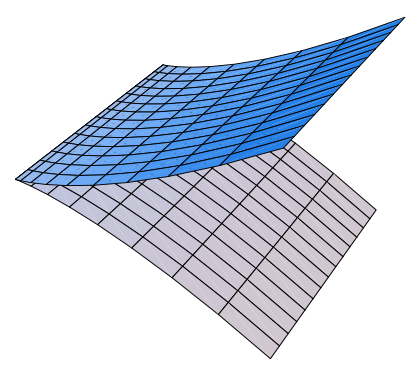

Figure 1: Cuspidal edge

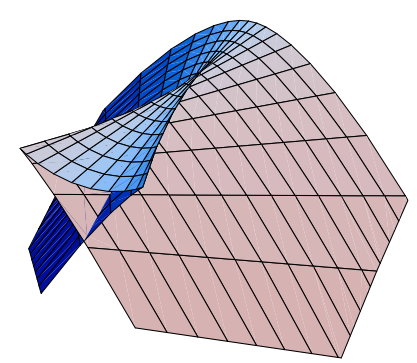

Figure 2: Swallowtail

The assertion of Theorem 8.1 can be interpreted as saying that the Legendrian lift $\mathcal{L}^{ \pm}$of the lightcone Gauss image $\mathbb{L}^{ \pm}$is Legendrian stable at each point. In this case, the lightcone Gauss image $\mathbb{L}^{ \pm}$has only cuspidal edges and swallowtails as singularities.

Corollary 8.2. Let $\mathcal{O} \subset \operatorname{Sp}-\operatorname{Emb}\left(U, S_{1}^{n}\right)$ be the same open dense subset as in Theorem 8.1. Let $\mathbf{X} \in \mathcal{O}, \mathbf{v}_{0}^{ \pm}=\mathbb{L}^{ \pm}\left(u_{0}\right)$ and $h_{\mathbf{v}_{0}^{ \pm}}:\left(U, u_{0}\right) \longrightarrow \mathbb{R}$ be the lightcone height function germ at $u_{0}$. Then we have the following.

(1) The point $u_{0}$ is an $L^{ \pm}$-parabolic point of $\mathbf{X}$ if and only if $H$-corank ${ }^{ \pm}\left(\mathbf{X}, u_{0}\right)=1$ (that is, $u_{0}$ is not an $L^{ \pm}$-flat point). In this case, $h_{\mathbf{v}_{0}^{ \pm}}$has the $\mathcal{A}_{k^{-}}$-type singularity for $k=2,3$.

(2) Suppose that $u_{0}$ is an $L^{ \pm}$-parabolic point of $\mathbf{X}$. Then the following conditions are equivalent:

(a) $\mathbb{L}^{ \pm}$has the cuspidal edge at $u_{0}$;

(b) $h_{\mathbf{v}_{0}^{ \pm}}$has the $\mathcal{A}_{2}$-type singularity;

(c) ${\mathrm{H}-\operatorname{ord}^{ \pm}}^{ \pm}\left(\mathbf{X}, u_{0}\right)=2$;

(d) The tangent de Sitter horospherical indicatrix germ is an ordinary cusp, where a curve $C \subset \mathbb{R}^{2}$ is called an ordinary cusp if it is diffeomorphic to the curve given by $\left\{\left(u_{1}, u_{2}\right) \mid u_{1}^{2}-u_{2}^{3}=0\right\}$

(3) Suppose that $u_{0}$ is an $L^{ \pm}$-parabolic point of $\mathbf{X}$. Then the following conditions are equivalent: 
(a) $\mathbb{L}^{ \pm}$has the swallowtail at $u_{0}$;

(b) $h_{\mathbf{v}_{0}^{ \pm}}$has the $\mathcal{A}_{3}$-type singularity;

(c) $\mathrm{H}-\operatorname{ord}^{ \pm}\left(\mathbf{X}, u_{0}\right)=3$;

(d) The tangent de Sitter horospherical indicatrix germ is a point or a tachnodal, where a curve $C \subset \mathbb{R}^{2}$ is called an tachnodal if it is diffeomorphic to the curve given by $\left\{\left(u_{1}, u_{2}\right) \mid u_{1}^{2}-u_{2}^{4}=0\right\}$

(e) For each $\varepsilon>0$, there exist $L^{ \pm}$-non-parabolic points $u_{1}, u_{2} \in U$ such that $\left\|u_{0}-u_{i}\right\|<$ $\varepsilon$ for $i=1,2$, and the tangent de Sitter horospheres to $M=\mathbf{X}(U)$ at $u_{1}$ and $u_{2}$ are equal.

Proof. Since $n=3, u_{0}$ is $L^{ \pm}$-parabolic point if and only if $\mathrm{H}$-corank ${ }^{ \pm}\left(\mathbf{X}, u_{0}\right) \geq 1$. By classification of singularities, $h_{\mathbf{v}_{0}^{ \pm}}$has only the $\mathcal{A}_{2}$ or $\mathcal{A}_{3}$-type singularities. We can avoid the case that $u_{0}$ is $L^{ \pm}$-flat point, so that $\mathrm{H}$-corank ${ }^{ \pm}\left(\mathbf{X}, u_{0}\right)=1$.

By Theorem 5.6, the conditions of (2) are equivalent. Similarly, the conditions (a),(b),(c),(d) of (3) are also equivalent. Suppose that corresponding Gauss image has swallowtail at $u_{0}$. We can observe that there is a self-intersection curve approaching $u_{0}$. (cf. Figure 2.) On this curve, there are two distinct points $u_{1}$ and $u_{2}$ such that $\mathbb{L}^{ \pm}\left(u_{1}\right)=\mathbb{L}^{ \pm}\left(u_{2}\right)$. By Lemma 5.2, this means that tangent de Sitter horospheres to $M=\mathbf{X}(U)$ at $u_{1}$ and $u_{2}$ are equal. On the other hand, if the Gauss image has cuspidal edge at $u_{0}$, there are no self-intersection on $\mathbb{L}^{ \pm}$. (cf. Figure 1.) This means that $(3)(\mathrm{a})$ is equivalent to $(3)(\mathrm{e})$. This completes the proof.

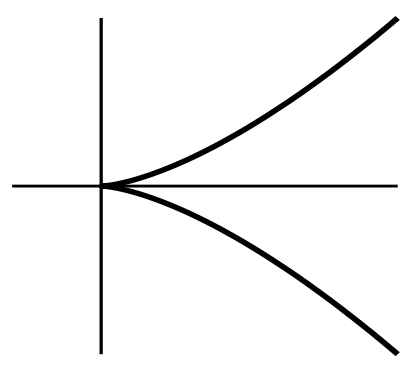

Figure 3: ordinary cusp

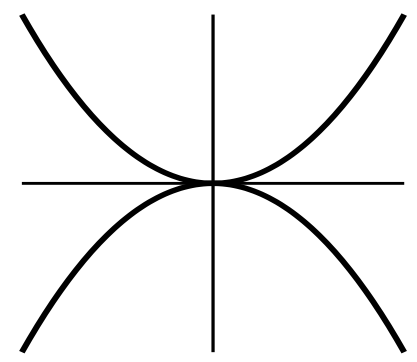

Figure 4: tachnodal

\section{Examples in de Sitter 3-space}

In this section we give some examples using the spacelike Monge form in de Sitter space.

Example 9.1. If $f\left(u_{1}, u_{2}\right)=\frac{1}{3} u_{1}^{3}+\frac{1}{2} u_{1}^{2}$, then

$$
\mathbf{X}_{f}\left(u_{1}, u_{2}\right)=\left(-\frac{1}{3} u_{1}^{3}-\frac{1}{2} u_{1}^{2},-\sqrt{1+\left(\frac{1}{3} u_{1}^{3}+\frac{1}{2} u_{1}^{2}\right)^{2}-u_{1}^{2}-u_{2}^{2}}, u_{1}, u_{2}\right)
$$


and $\kappa_{1}=1, \kappa_{2}=0$. Then we have $\bar{\kappa}_{1}^{+}(\mathbf{0})=0, \bar{\kappa}_{2}^{+}(\mathbf{0})=-1, \bar{\kappa}_{1}^{-}(\mathbf{0})=-2$ and $\bar{\kappa}_{2}^{-}(\mathbf{0})=-1$. So the origin is not $L^{-}$-parabolic point but a $L^{+}$-parabolic point. The positive tangent de Sitter horospherical indicatrix germ is the ordinary cusp $\left\{\left(u_{1}, u_{2}\right) \mid 2 u_{1}^{3}=3 u_{2}^{2}\right\}$. Therefore, the lightcone Gauss image $\mathbb{L}^{-}$is non-singular at the origin and $\mathbb{L}^{+}$is a cuspidal edge at the origin.

Example 9.2. If $f\left(u_{1}, u_{2}\right)=\frac{1}{2} u_{1}^{4}+\frac{1}{2} u_{1}^{2}$, then

$$
\mathbf{X}_{f}\left(u_{1}, u_{2}\right)=\left(-\frac{1}{2} u_{1}^{4}-\frac{1}{2} u_{1}^{2},-\sqrt{1+\left(\frac{1}{2} u_{1}^{4}+\frac{1}{2} u_{1}^{2}\right)^{2}-u_{1}^{2}-u_{2}^{2}}, u_{1}, u_{2}\right)
$$

and $\kappa_{1}=1, \kappa_{2}=0$. For the same reason as in the previous example, the origin is not $L^{-}$parabolic point but a $L^{+}$-parabolic point. The positive tangent de Sitter horospherical indicatrix germ is the tachnodal $\left\{\left(u_{1}, u_{2}\right) \mid u_{1}^{4}=u_{2}^{2}\right\}$. Therefore, the lightcone Gauss image $\mathbb{L}^{-}$is non-singular at the origin and $\mathbb{L}^{+}$is a swallowtail at the origin.

\section{Acknowledgment}

The author wishes to express his thanks to Professor Shyuichi Izumiya, Kentaro Saji and Takashi Sano for good advice and valuable discussions.

\section{References}

[1] V.I. Arnold, S.M. Gusein-Zade and A.N. Varchenko, Singularities of Differential Maps, Volume I, Birkhäuser, Basel, 1986.

[2] T. Banchoff, T. Gaffney and C. McCrory, Cusps of Gauss mappings, Research Notes in Mathematics 55, Pitman, London, 1982.

[3] D. Bleeker and L. Wilson, Stability of Gauss maps, Illinois J. Math. 22 (1978) 279-289.

[4] J. W. Bruce, The dual of generic hypersurfaces, Math. Scand. 49 (1981) 36-60.

[5] S. Izumiya, D. Pei and T. Sano, Singularities of hyperbolic Gauss maps, Proc. London Math Soc. 86 (2003) 485-512.

[6] E.E. Landis, Tangential singularities, Funct. Anal. Appl. 15 (1981) 103-114.

[7] J.A. Montaldi, On contact between submanifolds, Michigan Math. J. 33 (1986) 195-199.

[8] O.A. Platonova, Singularities in the problem of the quickest way round an obstruct, Funct. Anal. Appl. 15 (1981) 147-148.

[9] M.C. Romero-Fuster, Sphere stratifications and the Gauss map, Proc. Roy. Soc. Edinburgh Sect. A 95 (1983) 115-136. 
[10] G. Wassermann, Stability of Caustics, Math. Ann. 216 (1975) 43-50.

[11] V.M. Zakalyukin, Lagrangian and Legendrian singularities, Funct. Anal. Appl. 10 (1976) $26-36$.

[12] V.M. Zakalyukin, Reconstructions of fronts and caustics depending one parameter and versality of mappings, J. Soviet. Math. 27 (1984) 2713-2735.

Masaki Kasedou,

Department of Mathematics, Hokkaido University,

Sapporo 060-0810, Japan.

e-mail:kasedou@math.sci.hokudai.ac.jp 